\title{
Neurological Outcomes of Normothermia versus Targeted Temperature Management in Post-cardiac Arrest Syndrome
}

\author{
Havva Kocayigit ${ }^{1}$, Kezban Ozmen Suner ${ }^{2}$, Burak Kaya ${ }^{1}$, Yakup Tomak ${ }^{1}$, Ayça Taş Tuna ${ }^{1}$ and Ali Fuat Erdem ${ }^{1}$ \\ ${ }^{1}$ Department of Anaesthesiology and Reanimation, Sakarya University Education And Research Hospital, Sakarya, Turkey \\ ${ }^{2}$ Department of Intensive Care,Sakarya University Education And Research Hospital,Sakarya, Turkey \\ ${ }^{3}$ Department of Intensive Care, Sakarya University Education and Research Hospital, Sakarya, Turkey
}

\begin{abstract}
Objective: To determine the neurological outcome of targeted temperature management (TTM)for patients scoring lower than 8 on the Glasgow coma scale (GCS) upon return of spontaneous circulation (ROSC) after cardiac arrest.

Study Design: Retrospective cohort study.

Place and Duration of Study: Department of Anaesthesiology and Reanimation, Sakarya University Education And Research Hospital, Sakarya, Turkey from January 2018 to October 2019.

Methodology: Data of patients hospitalised in the Anaesthesialntensive Care unit for cardiac arrest receiving TTM and standard supportive therapy were analysed. Neurological outcome was evaluated with cerebral performance category (CPC) scores. Hospital stay and 30-day mortality was also noted.

Results: Data from 58 patients were analysed; 31 had received standard supportive therapy (non-TTM group) and 27 were treated with TTM (TTM group). There was no significant difference in hospital stay and patients' 30-day mortality between the two groups. The number of patients in the TTM group with CPC scores of 1 and 2, rated as a good neurological result, was significantly higher $(n=11,40.7 \%)$ than in the non-TTM group $(n=2,6.5 \% ; p=0.002)$. The number of patients with CPC scores of 3 and 4 , rated as having a neurological disability, was higher in the non-TTM group $(n=9,29 \%)$ than in the TTM group $(n=1,3.7 \%)$.

Conclusion: Neurological results were better in the TTM group of patients with ROSC. However, there was no significant difference in mortality between the TTMand non-TTM groups.
\end{abstract}

Key Words: Targeted temperature management, Cardiac arrest, Neurological outcome, Cerebral performance category.

How to cite this article: Kocayigit H, Suner KO, Kaya B, Tomak Y, Tuna AT, Erdem AF. Neurological Outcomes of Normothermia versus Targeted Temperature Management in Post-cardiac Arrest Syndrome. J Coll Physicians Surg Pak 2021; 31(05):497-501.

\section{INTRODUCTION}

Cardiac arrest is a major cause of mortality and neurological disability. The primary determinant of morbidity and mortality outcomesafter cardiac arrest is anoxic brain injury. ${ }^{1}$ During cardiac arrest, cessation of cerebral oxygen delivery occurs within minutes, with resultant neuron ischemia and cell death. The brain is devoid of nutrient stores, and consequently, neuroglycopenia and metabolic crisis occur within minutes after cardiac arrest, leading to cell death. ${ }^{2}$ Secondary injury is the additional cerebral injury characterised by an imbalance between cerebral oxygen delivery anddemandin the postresuscitation period, ultimately culminating in neuronal death. It begins immediately after the return of spontaneous circulation (ROSC).

Correspondence to: Dr. Havva Kocayigit, Department of Anaesthesiology and Reanimation, Sakarya University Education and Research Hospital, Sakarya, Turkey

E-mail: havvakocayigit@gmail.com

Received: January 06, 2021; Revised: March 26, 2021;

Accepted: April 20, 2021

DOI: https://doi.org/10.29271/jcpsp.2021.05.497
Structures, especially susceptible, include thehippocampi, thalami, cerebral cortex, corpus striatum, and cerebellar vermis, owing to metabolically high active tissue. Post-cardiac arrest syndrome includes anoxic brain injury, cardiac dysfunction, whole body ischemia, and reperfusion injury.Management of post-cardiac arrest syndrome focusses on limiting secondary injury by optimizing the balance between cerebral oxygen delivery and its use. ${ }^{3}$

At the cellular level, the beneficial effects of hypothermia are well documented on comatose survivors of cardiac arrest. Cerebral metabolism is reduced by $5 \%$ to $10 \%$ per $1^{\circ} \mathrm{C}$ decrease in core body temperature. In addition, global carbon dioxide production and oxygen consumption decline in proportion to reductions in core body temperature. ${ }^{4,5}$ By decreasing cerebral metabolism, hypothermia avoids excessive intracellular anaerobic metabolism, which increases lactate production. Hypothermia also improves cerebral glucose use and allows available cellular energy stores to be used for necessary cellular functions required for neuronal survival.

Hyperthermia is associated with numerous pathophysiologi- 
calsequelae that are potentially harmful after anoxic brain injury. Hyperthermia may increase blood-brain barrier permeability, leading to worsening cerebral oedema, intracranial pressure, and cerebral ischemia. ${ }^{6}$

The 2016 American Heart Association (AHA)guidelines and the International Liaison Committee on Resuscitation (ILCOR) recommend targeted temperature management (TTM) as the standard protocol for all patients with a Glasgow Coma Scale (GCS) lower than 8 after cardiac arrest. ${ }^{7,8}$

The aim of this study was to describe the outcome of applying TTMin patient with GCS less than 8, after cardiac arrest compared to standard supportive therapy.

\section{METHODOLOGY}

Following approval from the Ethics Committee, the data of patients hospitalised for cardiac arrest in the anaesthesia intensive care unit between January 2018 and December 2019 were analysed. Inclusion criteria specified adult patients aged 18-70 years with a GCS score less than 8 , whose spontaneous circulation had returned after cardiac arrest, and who were admitted through the emergency room or coronary care unit. Patients were excluded from the study if the cause of their arrest was other than myocardial infarction, if data such as waiting time for cardio pulmonary resuscitation (CPR) were missing, and if the patient died before the end of the TTM procedure.

For each patient, the following data were obtained upon admission: age, gender, the presenting rhythm (ventricular fibrillation (VF), asystole), APACHE score, time before starting CPR, CPR duration, Glasgowcoma scale, and serum lactate levels. During the intensive care follow-up, renal failure, presence of sepsis, finalGCS score, and the patient's final status were added.

Of 58 patients, who met the study criteria, 27 had therapeutic hypothermia therapy and 31 did not. The Arctic Sun ${ }^{\circledR}$ device (Medivance Corp., Louisville, KY) was used for TTM. During hypothermia, deep sedation and neuromuscular blockers were applied to patients undergoing TTM. TTM was applied as rapid cooling in $1-3$ hours with a target of $33^{\circ}-35^{\circ}$ Celsius, maintaining the core body temperature constant at $33^{\circ}-35^{\circ}$ for the next 24 hours, then re-warming $0.2^{\circ}-0.33^{\circ} \mathrm{C}$ per hour to reach $36.5^{\circ}-37.5^{\circ} \mathrm{C}$. Standard supportive therapy was applied to the non-TTM patients. Although TTM should be applied to all patients after cardiac arrest, it could not be applied to patients who were admitted to intensive care while the TTM device was in use.

The primary outcome was survival and hospital discharge with good neurological condition. Neurological status was evaluated using the cerebral performance category (CPC). CPC levels are scored as 1 = normal mental performance, $2=$ mild disability, $3=$ severe disability, $4=$ vegetative state, and $5=$ brain death. CPC points of 1 and 2 indicated a good neurological outcome, 3 to 5 points reflected poor neurological outcomes. ${ }^{9}$ The secondary outcome was the time to hospital discharge of the survivors.

\section{RESULTS}

Sixty-nineconsecutive patients with ROSC following myocardial infarction who were admitted to the ICU between January 2018 and December 2019. Eight patients were excluded because they died within the first 24 hours; and three patients had multiple cardiac arrests. Thus, 58 patients were included in the analysis. Of those, 31 (53.4\%) patients had received standard supportive therapy (non-TTM group) and27 (46.6\%) patients had TTM (TTM group).

The mean age of patients was $56.4 \pm 8.4$ years in the TTM group and $59.8 \pm 8.0$ years in the non-TTM group. In the TTM group, $85.2 \%$ of the patients were males, and in the non-TTM group, $87.1 \%$ were males. Hypertension (10 [32.3\%] vs. 4 [14.8\%]), diabetes mellitus (8 [25.8\%] vs.3 [11.1\%]), and coronary artery disease (8 [25.8\%] vs.6[22.2\%]) were the mostcommon comorbidities (Tablel).

There was a statistically significant difference between the APACHE scores of the two groups $(34.7 \pm 3.7$ versus $27.5 \pm 7.6$, $p<0.001)$. The number of patients with witnessed arrest in the TTM group,18 (66.7\%), was significantly higher than in the nonTTM group, $7(22.6 \%)(p=0.001)$. However, there were no statistically significant differences in out-of-hospital cardiac arrest rates, presenting shockable rhythm, duration of CPR, inotropic agent infusion at admission, serum lactate levels, and development of acute renal failure between the TTM group and the nonTTM group $(p=0.217, p=0.820, p=0.426, p=0.268, p=0.160$, and $p=0.092$, respectively) (Table I).

There was no significant difference between the two groups in length of hospital stay $(p=0.074)$ and 30-day mortality $(p=$ 0.847). Patients who were not deceased were evaluated as "survivors," and there was no difference in survival rates between the TTM group and the non-TTM group (11 [35.5\%] versus 14 [51.9\%], respectively, $p=0.209$ ). There was a significant difference between the neurological status of the survivors in the TTM group and the non-TTM group. Neurological outcome was evaluated with the CPC score.The number of patients with CPC scores of 1 and 2 , defined as a good neurological result, was significantly higher in the TTM groupthan in the non- $T$ M group (11 [40.7\%] vs. 2 [6.5\%], respectively, $p=0.002$ ). Patients with CPC scores of 3 and 4 were evaluatedas having a neurological disability. The number of patients with CPC scores of 3 and 4 in the non-TTM group was higherthan in the TTM group ( $n=9,29 \%$, and $n=1,3.7 \%$, respectively) (Figure 1 ). It was found that a significant difference between the time from hospitalisation to discharge among survivors in the two groups (87.2 $\pm 33.1 \mathrm{vs}$. $43.1 \pm 35.5, p=0.039$ ) (Table II).

\section{DISCUSSION}

In this observational study, the clinical characteristics, disease severity, and clinical outcomes of TTM versus normothermia were investigated. It was observed that TTM was associated with better neurological outcomes and shortened the hospitalisation period (among the survivors), but did not affect 30-day mortality and survival. 
Table I: Baseline characteristics and disease severity.

\begin{tabular}{|c|c|c|c|}
\hline & $\begin{array}{l}\text { Non-TTM } \\
(n=31)\end{array}$ & $\begin{array}{l}\text { TTM } \\
(n=27)\end{array}$ & $p$ \\
\hline Age (years) & $59.8 \pm 8.0$ & $56.4 \pm 8.4$ & 0.113 \\
\hline $\begin{array}{c}\text { Gender } \\
\text { Male } \\
\text { Female }\end{array}$ & $\begin{array}{l}(n, \%) \\
27,87.1 \\
4,12.9\end{array}$ & $\begin{array}{l}(n, \%) \\
23,85.2 \\
4,14.8\end{array}$ & $\begin{array}{l}p \\
>0.999\end{array}$ \\
\hline $\begin{array}{c}\text { Comorbidities } \\
\text { HT } \\
\text { DM } \\
\text { CAD } \\
\text { Malignancy }\end{array}$ & $\begin{array}{l}(\boldsymbol{n}, \%) \\
10,32.3 \\
8,25.8 \\
8,25.8 \\
0\end{array}$ & $\begin{array}{l}(n, \%) \\
4,14.8 \\
3,11.1 \\
6,22.2 \\
2,7.4 \\
\end{array}$ & $\begin{array}{l}p \\
0.121 \\
0.154 \\
0.750 \\
0.212 \\
\end{array}$ \\
\hline APACHE Score & $34.7 \pm 3.7$ & $27.5 \pm 7.6$ & $<0.001$ \\
\hline Witnesses to arrest & $7,22.6$ & $18,66.7$ & 0.001 \\
\hline $\begin{array}{l}\text { Cardiac arrest } \\
\text { Out of hospital } \\
\text { in hospital }\end{array}$ & $\begin{array}{l}(n, \%) \\
21,67.7 \\
10,32.3\end{array}$ & $\begin{array}{l}(n, \%) \\
14,51.9 \\
13,48.1\end{array}$ & $\begin{array}{l}p \\
0.217\end{array}$ \\
\hline Presenting shockable rhythm (VF/VT) & $\begin{array}{l}(n, \%) \\
14,45.2\end{array}$ & $\begin{array}{l}(n, \%) \\
13,48.1\end{array}$ & 0.820 \\
\hline Duration of CPR, median (IQR) & $\begin{array}{l}(\min ) \\
20(15-30)\end{array}$ & $\begin{array}{l}(\min ) \\
20(10-35)\end{array}$ & 0.426 \\
\hline Inotropic agent infusion & $\begin{array}{l}(n, \%) \\
20,64.5\end{array}$ & $\begin{array}{l}(n, \%) \\
21,77.8\end{array}$ & 0.268 \\
\hline Lactate (mmol/L), median (IQR) & $6.8(4.3-8.8)$ & $4.2(1.6-10.2)$ & 0.160 \\
\hline Acute renal failure & $\begin{array}{l}(n, \%) \\
12,38.7\end{array}$ & $\begin{array}{l}(n, \%) \\
5,18.5\end{array}$ & 0.092 \\
\hline
\end{tabular}

Table II: Outcomes.

\begin{tabular}{|c|c|c|c|}
\hline & Non-TTM $(n=31)$ & TTM $(n=27)$ & $p$ \\
\hline Hospital stay (days), median (IQR) & $39(10-69)$ & $17(4-29)$ & 0.074 \\
\hline 30 days mortality $(n, \%)$ & $13,41.9$ & $12,44.4$ & 0.847 \\
\hline Survival $(n, \%)$ & $11,35.5$ & $14,51.9$ & 0.209 \\
\hline Hospital stay before discharge, (days) & $87.2 \pm 33.1$ & $43.1 \pm 35.5$ & $0.004 *$ \\
\hline Good neurologic results $(n, \%)$ & $2,6.5)$ & $11,40.7$ & $0.002 *$ \\
\hline
\end{tabular}

Although significant differences between mortality and 30day mortality between the two groups were not found, there are many studies in the literature reporting that TTM decreases mortality. In a retrospective cohort study examining patients with out-of-hospital cardiac arrest, the mortality of the TTM group was found to be significantly lower than that of the non-treated group. ${ }^{9}$ The FINNRESUSCI study that examined non-hospital cardiac arrest patients reported that $\Pi \mathrm{TM}$ application reduced mortality. ${ }^{10}$ In addition, in a review published in 2016 of non-hospital cardiac arrest patients, treatment with TTM after ROSC was shown to reduce mortality (OR $0.51 ; 95 \% \mathrm{Cl} 0.40-0.64) .{ }^{11}$

Researchers recently published a large randomised controlled trial involving 950 patients who were unconscious after cardiac arrest. They stated that there was no difference in mortality between $33^{\circ}$ and $36^{\circ}$ target temperature $\left(33^{\circ} \mathrm{C}\right.$, $50 \%$ and $36^{\circ} \mathrm{C}, 48 \%$ ) as in this study. ${ }^{12}$ Further, in a study involving 950 unconscious survivors of out-of-hospital cardiac arrest, alarge meta-analysis stated that TTM treatment had no effect on mortality. ${ }^{13,14}$ In the literature, although concensus on the effect of TTMon mortality has not yet been reached, a difference between the two groups in mortality was not found.

In previous studies, the neurological status of patients was generally evaluated using the classification of cerebral performance category. Patients with a score of 1 and 2 were accepted as having good neurological outcomes. ${ }^{15}$ In this study, compatible with the literature, the authorsevaluated patients with CPC scores 1 and 2 as having good neurological status and found that the number of patients with good neurological status was significantly higher in the TTM group $(40.7 \%)$ than in the non-TTM group $(6.5 \%, p=0.002)$. According to a Cochrane analysis first published in 2009 and updated over the years, participants in the conventional cooling group were more likely to achieve a favourable neurological outcome than the non-cooling group (risk ratio [RR] 1.94, 95\% confidence interval [CI] 1.18 to 3.21$).{ }^{16}$ Therefore, we think that TTM application reduces the disability level of patients by having a neuroprotective effect.

In this study, the number of surviving patients was similar in the two groups, but comparing the duration of hospitalisation of survivors reflected that the non-TTM group stayed in the hospital longer than the TTM group before discharge $(p=0.004)$. Survivors in the non-TTM group mostly have CPC 
scores of 3 and 4 , which indicates severe disability. Before discharge, patients with severe disabilities need some surgical procedures, such as tracheostomy and percutaneous endoscopic gastrostomy, and require a home ventilator. In this regard, we think that TTM application influences the time of hospital discharge in relation to the level of neurological disability.

Although there is no age limit in the literature for TTM application, the general approach is to apply TTM for younger patients. A study on the effectiveness of TTM in patients aged 75 and over indicated that TTM does not have a positive effect on CPC scores and mortality. ${ }^{17}$ Similarly, a study investigating the effectiveness of TTM in elderly patients found no statistically significant difference in neurological outcomes and mortality between patients having $a 33^{\circ}$ and a $36^{\circ}$ target temperature. ${ }^{18}$ In our daily practice, we evaluate TTM application based on the premise that neurological recovery decreases with age. To collect data for analysis in this study, older patients were excluded due to the high risk of unfavourable outcomes in patients over 75 years.

Different methods used for cooling have proven to be effective. These include surface cooling methods (pre-cooled surface cooling pads, water-circulating surface cooling pads) and intra-body cooling methods (cooled intravenous fluids, endovascular devices inserted through a central catheter). ${ }^{19,20}$ However, external water-circulating cooling pads with gel coating are easy to apply for reliable temperature control. In our study, TTM was applied with the Arctic Sun ${ }^{\circledR}$ device (Medivance Corp., Louisville, KY).

This retrospective study has the usual limitations of retrospective studies, such as randomisation. Due to the study design, we could not obtain data of door-to-cooling time or the time to reach the targeted temperature for patients on whom TTM was applied.

\section{CONCLUSION}

This study found that neurological results were better in the TTM group in patients with ROSC, in accordance with the literature. Among the survivors in the non-TTM group, the hospital stay was longer due to poor neurological results. However, there was no difference in mortality between the two groups.

\section{ETHICAL APPROVAL:}

The study was approved by Sakarya University Education and Research Hospital, Clinical Research Ethics Committee. (Approval date and number: 26.02.2020/337).

\section{PATIENTS' CONSENT:}

All patients signed a document of informed consent.

\section{CONFLICT OF INTEREST:}

The authors declared no conflict of interest.
HK: Designed, directed and coordinated the study, created study plan, analysed the data and written the article.

KÖS, BK,YT: Selected patients and collected samples and analysed the data.

ATT, AFE: Studied the collected samples in biochemistry laboratory.

\section{REFERENCES}

1. Stub D, Bernard S, Duffy SJ, Kaye DM. Post-cardiac arrest syndrome: A review of therapeutic strategies. Circulation 2011;123(13):1428-35.

2. Wagner SR 4th, Lanier WL. Metabolism of glucose, glycogen, and high-energy phosphates during complete cerebral ischemia. A comparison of normoglycemic, chronically hyperglycemic diabetic, and acutely hyperglycemic nondiabetic rats. Anesthesiology 1994;81(6):1516-26. doi: 10.1097/00000542-199412000-00028.

3. Nolan JP, Neumar RW, Adrie C. Post-cardiac arrest syndrome: Epidemiology, pathophysiology, treatment, and prognostication. A scientific statement from the international liaison committee on resuscitation; the American Heart Association Emergency Cardiovascular Care Committee; the council on cardiovascular surgery and anesthesia; the council on cardiopulmonary, perioperative, and critical care; the council on clinical cardiology; the council on stroke. Resuscitation 2008;79(3):350-79. doi: 10.1016/j.resuscitation.2008.09.017.

4. Polderman KH. Induced hypothermia and fever control for prevention and treatment of neurological injuries. Lancet 2008;371:1955-69. doi: 10.1016/S0140-6736(08)60837-5.

5. Polderman KH. Mechanisms of action, physiological effects, and complications of hypothermia. Crit Care Med2009;37(7 Suppl):S186-202.doi: 10.1097/CCM.0b013e3181aa5241.

6. Oh JY, Jo K, Joo W, Yoo DS, Park H. Temperature difference between brain and axilla according to body temperature in the patient with brain injury. Korean J Neurotrauma 2020;16(2):147-56. doi: 10.13004/kjnt.2020.16.e40.

7. Donnino MW, Andersen LW, Berg KM, Reynolds JC, Nolan P, Morley PT, et al. Temperature management after cardiac arrest: An advisory statement by the advanced life support task force of the international liaison committee on resuscitation and the american heart association emergency cardiovascular care committee and the council on cardiopulmonary, critical care, perioperative and resuscitation. Resuscitation 2016; 98:97-104.doi: 10.1016/j.resuscitation.2015. 09.396.

8. Callaway CW, Donnino MW, Fink EL, Geocadin RG, Golan E,Kern KB, et al. Part 8: post-cardiac arrest care: 2015 American heart association guidelines update for cardiopulmonary resuscitation and emergency cardiovascular care. Circulation 2015;132(18 Suppl 2):S465-82. doi: 10.1161/CIR.0000000000000262.

9. Ko PY, Wang LL, Chou YJ, JP Tsai J, Huang SH, Chang CP, et al.Usefulness of therapeutic hypothermia to improve survival in out-of-hospital cardiac arrest. ActaCardiol $\operatorname{Sin} 2019 ; 35(4): 394-401$. doi: 10.6515/ACS.201907_35(4). 20190113A.

10. Vaahersalo J, Hiltunen $\mathrm{P}$, Tiainen $\mathrm{M}$, Oksanen $\mathrm{T}$, Kaukonen

\section{AUTHORS' CONTRIBUTION:}


KM, Pettilä V. Therapeutic hypothermia after out-of-hospital cardiac arrest in Finnish intensive care units: The FINNRESUSCI study. Intensive Care Med 013; 39(5):826-837. doi: 10.1007/s00134-013-2868-1.

11. Schenone AL, Cohen A, Patarroyo G, Harper L, Wang $\mathrm{X}$, Shishehbor $\mathrm{MH}$, et al. Therapeutic hypothermia after cardiac arrest: A systematic review/meta-analysis exploring the impact of expanded criteria and targeted temperature. Resuscitation2016;108:102-110. doi: 10.1016/j.resuscitation.2016.07.238.

12. Nielsen N, Wetterslev J, Cronberg T, Erlinge D, Gasche $Y$, Hassager $\mathrm{C}$, et al. Targeted temperature management at $331 \mathrm{C}$ versus $361 \mathrm{C}$ after cardiac arrest. N Engl J Med 2013; 369:2197-206.

13. Granberg B, McGillis E, Solbiati M, Gruppo di AutoformazioneMetodologica (GrAM). Therapeutic induced hypothermia does not improve the prognosis of out-of-hospital cardiac arrest patients. Intern Emerg Med 2014; 9(6):677-9. doi: 10.1007/s11739-014-1100-9.

14. Hakim SM, AmmarMA, Reyad MS. Effect of therapeutic hypothermia on survival and neurological outcome in adults suffering cardiac arrest: a systematic review and meta-analysis. Minerva Anestesiol 2018; 84(6):720-30. doi: 10.23736/S0375-9393.18.12164-X.

15. Edgren E, Hedstrand U, Kelsey S, Sutton-Tyrrell K, Safar P. Assessment of neurological prognosis in comatose survivors of cardiac arrest. BRCT I study group. Lancet 1994; 343(8905):1055-9.doi: 10.1016/s0140- 6736(94) 90179-1.

16. Arrich J, Holzer M, Havel C, Müllner M, Herkner H. Hypothermia for neuroprotection in adults after cardiopulmonary resuscitation. Cochrane Database Syst Rev 2009;4:CD004128.doi: 10.1002/14651858. CD004 128.pub2.

17. Mader TJ, Westafer LM, Nathanson BH, Villarroel N, Coute RA, McNally BF. Targeted temperature management effectiveness in the elderly: Insights from a large registry. Ther Hypothermia Temp Manag2017;7(4):222-30. doi: 10.1089/ther.2017.0015.

18. Winther-Jensen M, Pellis T, Kuiper M, Koopmans M, Hassager $\mathrm{C}$, Nielsen $\mathrm{N}$, et al. Mortality and neurological outcome in the elderly after target temperature management for outof-hospital cardiac arrest. Resuscitation2015;91:92-8. doi: 10.1016/j.resuscitation.2014.12.030.

19. Wu L, Huber M, Wu D, Chen J, Li M, Ding Y, et al. Intra-arterial Cold Saline Infusion in Stroke: Historical Evolution and Future Prospects. Aging Dis 2020;11(6):1527-36. doi: 10.14336/AD.2020.0325.

20. Smith T, Couillard P, McBeth P, Hruska P, Kortbeek J. Esophageal cooling for hypoxic Ischemic encephalopathy: A feasibility study. Ther Hypothermia Temp Manag2020; doi: 10.1089/ther.2020.0015. 